\title{
Nuevas aportaciones a la gestión informatizada de derrames de hidrocarburos: su aplicación a "zonas refugio"
}

\author{
J.R. Bergueiro ${ }^{1 *}$, S. Moreno ${ }^{1}$, L. Hernández ${ }^{1}$, J.M. Calvilla², J.I. Gómez ${ }^{2}$ y J. González ${ }^{2}$ \\ ${ }^{1}$ Universidad de las Islas Baleares. Facultad de Ciencias. Carretera de Valldemossa Km. 7.5, \\ 07122 Palma de Mallorca. España \\ E-mail: ramon.bergueiro@uib.es \\ ${ }^{2}$ Universidad de La Laguna. Escuela Técnica Superior de Náutica, Máquinas y Radioelectrónica Naval. \\ Vía Auxiliar Paso Alto s/n. 38001 Santa Cruz de Tenerife. Islas Canarias. España
}

(recibido/received: 28-Enero-2011; aceptado/accepted: 20-Mayo-2011)

\begin{abstract}
RESUMEN
El objeto del presente trabajo es el de describir la mayor parte de los procedimientos a seguir para decidir si es aconsejable trasladar un petrolero siniestrado a una "zona refugio". Para tomar esta decisión se deberán considerar diferentes procedimientos, con la finalidad de objetivizar al máximo dicha decisión. Es de gran importancia conocer muy bien las características y las infraestructuras de las diferentes zonas costeras que pueden ser consideradas posibles "zonas refugio". En base a todo lo anterior, se ha desarrollado un programa formado por varios módulos que permiten obtener datos de gran importancia, entre los que se incluyen las matrices de valores ambientales e infraestructurales, y que permite el cálculo de determinados factores determinantes para las actuaciones de traslado y preparación del petrolero (tiempo de traslado del petrolero, tiempo de atraque y conexión a los sistemas de bombeo, tiempo de vaciado de los hidrocarburos a los depósitos de almacenamiento, trasvase a otro petrolero, etc.). Además, dicho programa permite estimar los costes de limpieza y restauración, así como las pérdidas en el sector turístico.
\end{abstract}

Palabras claves: Hidrocarburos; Zona refugio; Derrames de hidrocarburos

\begin{abstract}
The purpose of this paper is to describe most of the procedures to follow in deciding whether you should transfer a tanker wrecked in a "refuge zone". In reaching this decision, it should consider different procedures, in order to objectify the best decision. It is important to know very well the features and infrastructures of the different coastal areas, which can be considered as possible "refuge zones". Based on the foregoing analysis, it has developed a program consisting of several modules to permit compute data of great importance, among which include matrices of environmental and infrastructural values that allow for the calculation of certain factors for transfer actions and preparation of the tanker (transfer time of the tanker, berthing time and connection time to the pumping systems, time of emptying the oil in storage tanks, transfer to another tanker, etc). In addition, this program allows us to estimate the costs of cleaning and restoration, as well as losses in the tourism sector.
\end{abstract}

Keywords: Hydrocarbons; Place of refuge; Oil spills

*Autor para la correspondencia 


\section{J.R. Bergueiro et al.}

\section{INTRODUCCIÓN}

Con aproximadamente 1.200 kilómetros de costas, las Islas Baleares (España), junto con las otras grandes islas del Mediterráneo Occidental, Córcega, Cerdeña y Sicilia, presentan un patrimonio único en términos de ecosistemas que debe preservarse en términos de biodiversidad.

Desde este punto de vistas estas islas pueden considerarse como "islas laboratorio" en donde la biodiversidad fue mantenida hasta la década de los 60 debido a una población relativamente baja y a una ausencia de turismo y de complejos industriales.

A partir de la década de los 60 las Islas Baleares tienen un desarrollo muy importante del turismo, recibiendo del orden de 9 millones de turistas frente a los 850.00 habitantes residentes.

La presencia de las diferentes actividades ligadas al turismo hace que el mar balear necesite tener un Plan de Gestión Integrada que permita reducir y gestionar los vertidos de contaminantes al mar.

Es importante resaltar que los ingresos por turismo en España superan los 18.000 millones de Euros y da ocupación a un millón y medio de personas incluyendo los puestos directos e indirectos.

Por otra parte las Islas Baleares se encuentran en una zona por donde transitan grandes petroleros transportando crudo de petróleo hasta las refinerías de Tarragona, Castellón de la Plana y Cartagena, todas ellas situadas en el levante español. Estos petroleros proceden de Libia y del Golfo Pérsico, vía Canal de Suez y vía Estrecho de Gibraltar. Igualmente por las inmediaciones de las Islas Baleares navegan petroleros transportando derivados del petróleo, tales como gasolinas, gasoil, querosenos de aviación y fueloil.

Cualquier accidente de un petrolero, en las inmediaciones de las Islas Baleares, puede originar un gran vertido de hidrocarburos con una gran repercusión en el sector turístico.
De acuerdo con las directrices dadas por la Organización Marítima Internacional (OMI) una "zona refugio" debe entenderse como: "un lugar a donde puede ir un buque necesitado de asistencia y pueda adoptar las medidas que le permitan estabilizar su situación y reducir los peligros para la navegación, y la protección de la vida humana y el medio ambiente" (OMI, Resolution A.949(23)). De lo anteriormente reseñado se deduce que una zona refugio debe ser entendida en el sentido más amplio posible ya que debe incluir puertos, bahías, calas y cualquier otro espacio que se encuentre bajo jurisdicción del estado ribereño, tal como lo establece el artículo 2.1 de la Convención de Naciones Unidas sobre el Derecho del Mar (CONDEMAR).

Cuando se produce un accidente de un petrolero $\mathrm{y}$ se encuentren en peligro vidas humanas los estados ribereños tienen la obligación de auxiliar al buque siniestrado, de acuerdo con las convenciones CONDEMAR y SERVIMAR (seguridad de la vida humana en el mar). Sin embargo, no ocurre lo mismo cuando no haya en peligro vidas humanas y sí haya un gran riesgo de contaminación por hidrocarburos de las costas de los estados limítrofes.

En este caso surge la controversia entre la necesidad de auxiliar al buque siniestrado, llevándolo a una zona refugio y el de proteger las costas de la posible contaminación por los hidrocarburos derramados originada por un buque que se encuentre en dificultades graves.

Hay que tener en cuenta que el artículo 11 del Convenio Internacional sobre Salvamento Marítimo establece que "un Estado Parte deberá, siempre que regule o decida sobre asuntos relativos a operaciones de salvamento tales como la admisión en puerto de buques en peligro proporcionar medios a los salvadores, tener en cuenta la necesidad de cooperación entre salvadores, otras partes interesadas y autoridades públicas para asegurar la eficacia y el éxito de las operaciones de salvamento para el propósito de salvar vidas o propiedades en peligro, así como para prevenir daños al medio ambiente en general". 
Algunos Estados que firmaron este Convenido no habían adoptado esta regulación, tal como se puso de manifiesto en los accidentes de los buques M/T ERIKA (Diciembre 1999), M/T CASTOR (Diciembre 2000) y M/T PRESTIGE (Noviembre 2002) a los que se les negó la entrada a zonas refugio.

\section{DESARROLLO DEL ESTUDIO}

La mayoría de los estudios sobre de vertidos de hidrocarburos en el mar abordan, mediante modelos de simulación, todo lo referente al desplazamiento de los hidrocarburos, a la trayectoria seguida, a la variación con el tiempo de las propiedades físicas de la mezcla remante y a las dimensiones de dicho vertido, pero no suelen tratan el posible traslado de un petrolero siniestrado a una zona refugio. Para poder decidir la conveniencia o no de trasladar un petrolero siniestrado a una zona refugio se ha desarrollado el modelo "REFUGIO" (Bergueiro y Domínguez, 1997). Dicho modelo consta de dos módulos, el primero de ellos permite la clasificación de las zonas costeras en función de su idoneidad como zonas refugio. El segundo de los módulos permite la estimación de los tiempos de determinadas actividades relacionadas con el traslado del buque siniestrado y el trasvase de su carga.

Para la clasificación de las posibles zonas refugio se ha desarrollado una escala de 28 puntos en la que la valoración mínima corresponde a una zona que por sus características nunca podría considerarse como zona refugio, por no reunir el mínimo de infraestructuras necesarias. La valoración máxima corresponde a una zona refugio que dispondría de todo el equipamiento anticontaminación necesario; es decir que estaría alejada de zonas habitadas, de zonas de pesca, de zonas sensibles y de zonas de tráfico marítimo, que dispondría de los medios necesarios para trasvasar los hidrocarburos y con posibilidad de desplazar a esa zona equipos para reparar el buque siniestrado, en el caso de que fuese necesario (Bergueiro et al., 2004).

La escala desarrollada tiene en cuenta los siguientes factores: equipación anticontaminación; cercanía a zonas habitadas, a zonas de pesca, a zonas sensibles y zonas de tráfico marítimo; posibilidad de desplazamiento de equipos; posibilidad del trasvase de la carga; posibilidad de quemar la carga en caso de ser necesario y riesgo de explosión.

Para poder aplicar correctamente la escala desarrollada, es necesario contar con toda una serie de informaciones de cada una de las zonas costeras. Con la finalidad de poder objetivizar y tratar dicha información, se han desarrollado dos tipos de matrices. La primera la integran factores ambientales y la segunda factores estructurales. Atendiendo a la información contenida en las mismas, así como a las características particulares que pueda tener cada una de las zonas, y de acuerdo a la interacción de estos datos con la escala desarrollada, es posible dar una valoración objetiva de la idoneidad de una determinada costa para actuar como zona refugio.

La matriz de factores ambientales está formada por 28 filas y 10 columnas. En las filas aparecen los procesos sufridos por los hidrocarburos derramados y las tareas que pueden desarrollarse durante las actuaciones anticontaminación. En las columnas aparecen los factores ambientales que se consideran susceptibles de ser afectados por los procesos y las actuaciones (batimetría, flora y fauna, zonas pesqueras, zonas sensibles, etc.).

En el caso de la matriz de factores estructurales, la misma tiene 28 filas y 13 columnas, siendo las filas las mismas que para la matriz ambiental y apareciendo en las columnas los factores estructurales que pueden estar presentes en una zona costera (estructuras artificiales, puertos deportivos, astilleros, hoteles, etc.) y que pueden verse afectados por diferentes factores de un vertido de hidrocarburos. Dichas matrices se han desarrollado para cada una de las zonas costeras de estudio y para diversos tipos de hidrocarburos (gasoils, Jet A1, JP8, fueloils y gasolinas).

Igualmente, es de gran importancia conocer los tiempos que supondrán determinadas tareas a desarrollar, tanto en el caso de que se decida llevar el petrolero siniestrado a una zona refugio, 
como si la decisión es no llevarlo y transvasar su carga a otro buque o, en el caso de estar muy cercano a la costa, trasvasar su carga a camiones cisterna, tal como se efectuó en el siniestro del petrolero Mar Egeo, en las inmediaciones de la Torre de Hércules en A Coruña (Bergueiro, 1993).

Teniendo en cuenta todo lo anterior, aplicando el módulo RESCATE, perteneciente al modelo REFUGIO, se pueden estimar el tiempo de traslado de un petrolero siniestrado a una zona refugio, el tiempo de atraque y conexión de los tubos distribuidores, el tiempo de vaciado del buque a una instalación portuaria y el tiempo de trasvase a otro buque, tomando como datos de partida: tonelaje del buque siniestrado, cantidad y tipo de combustible a bombear, distancia a la zona refugio, estado de la mar, tipo de traslado (por sus propios medios o mediante remolcadores) y lugar de bombeo (instalación portuaria, de buque a buque o de buque a camiones cisterna).

Cabe destacar que el modelo REFUGIO y todos los módulos que lo integran fueron desarrollados en Visual Basic 6.0.

\section{RESULTADOS Y DISCUSIÓN}

Existe siempre una gran controversia a la hora de decidir si debe llevarse o no un petrolero siniestrado a una zona refugio, debido a la gran complejidad que presenta dicho traslado y a los riesgos que puede originar dicho hecho. Por todo esto, es necesario disponer de herramientas que ayuden en la toma de decisiones con cierta objetividad. Para este fin, se han clasificado la mayor parte de las zonas costeras de la isla de Mallorca (245). Dicha isla se encuentra ubicada en el Archipiélago de las Islas Baleares (España), siendo la misma la de mayor extensión. Se caracteriza por el hecho de que la principal actividad económica se encuentra centrada en el turismo de playa, hecho que hace de gran importancia que el estado de la costa sea adecuado para poder mantener su economía.

Al estudiar el entorno costero, se pueden diferenciar zonas de características muy dispares, ya que pueden encontrarse desde playas de arena fina hasta zonas acantiladas. En base a la orografía costera, a la facilidad de comunicación y a las infraestructuras ubicadas en cada una de ellas y en sus cercanías (Ministerio de Medio Ambiente, Guía de playas), se han clasificado las diferentes ubicaciones con la finalidad de valorar su potencialidad como posibles zonas refugio.

Como ejemplo de la aplicación de las matrices de factores ambientales y estructurales se muestra una parte de las referentes a la playa de Albercutx (Pollensa), lugar de Interés Comunitario (Normativa Europea RED NATURA 2000-LIC), con alta ocupación turística y en cuya proximidad existe la zona húmeda de La Gola. La Fig. 1 muestra las primeras 15 filas de la matriz representativa de factores ambientales y la Fig. 2 las 15 primeras filas de la matriz de factores estructurales. La valoración de esta zona como posible zona refugio es muy baja, por lo que al analizar las zonas cercanas se observa que la mejor opción es el Puerto de Alcudia, por las infraestructuras con las que cuenta, siempre que se trate de un combustible sin riesgo de explosión.

Determinada la zona costera más idónea para el traslado a la misma del buque siniestrado, en función de su tonelaje, de las condiciones meteorológicas reinantes, del volumen y tipo de combustible a bombear y de la distancia a dicha zona, el modelo calcula el tiempo de traslado del petrolero, tanto si se desplaza por sus propios medios como si se desplaza arrastrado por remolcadores, el tiempo de atraque y conexión a los brazos de descarga y el tiempo de vaciado de los hidrocarburos a los depósitos de almacenamiento.

La Fig. 3 muestra una de las pantallas de entrada y salida de datos para una simulación del traslado de un petrolero, de menos de 35.000 toneladas (tonelaje de los petroleros que abastecen normalmente de combustible a las Islas Baleares) que transporte de $30.000 \mathrm{~m} 3 \mathrm{de}$ Gas Oil, situado a unas 5 millas de la zona refugio seleccionada, a la que puede desplazarse por sus propios medios, bajo unas condiciones meteorológicas de marejadilla. 


\section{J.R. Bergueiro et al.}

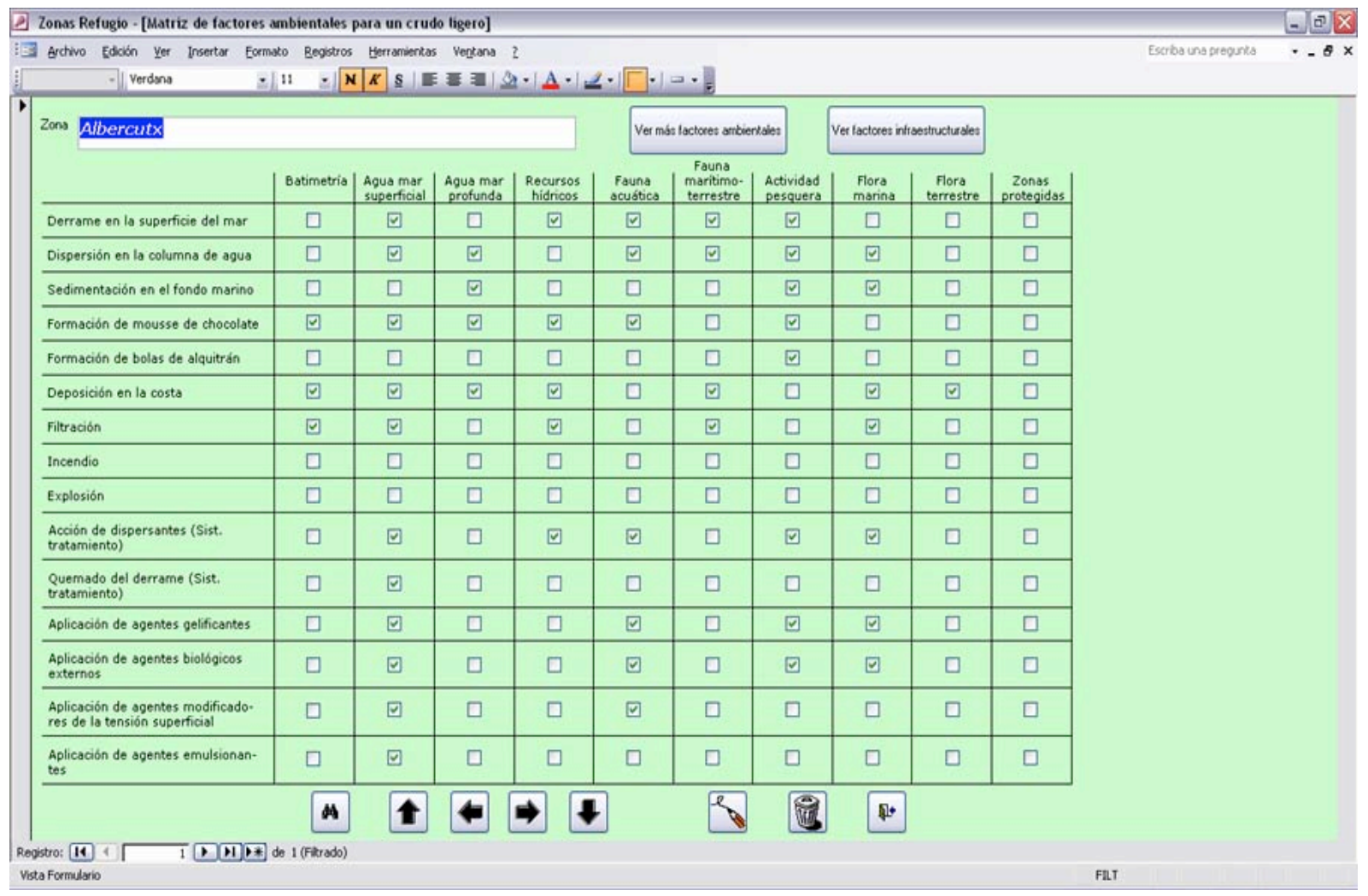

Fig. 1 Matriz de factores ambientales para un crudo de petróleo tipo ligero.

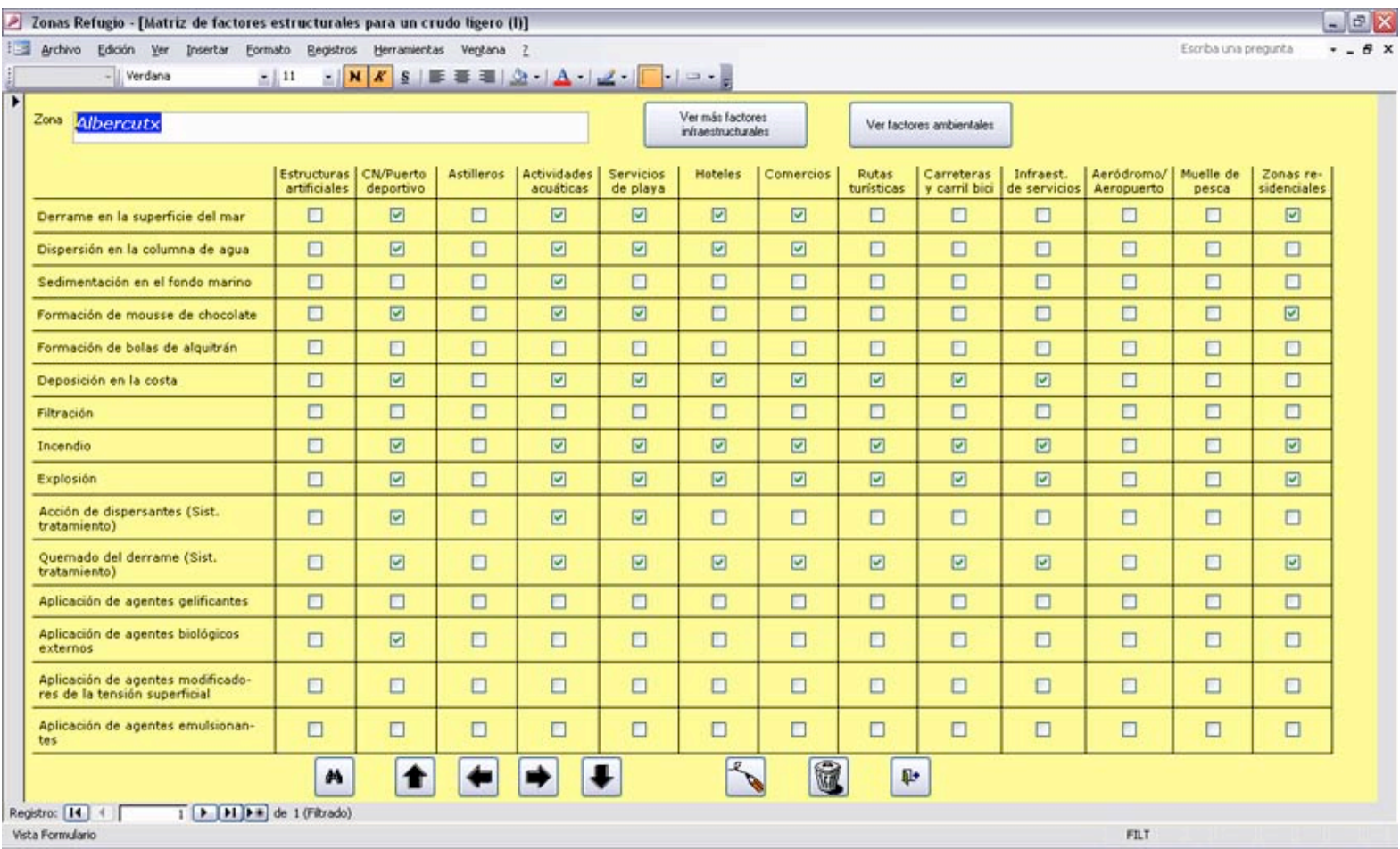

Fig. 2 Matriz de factores estructurales para un crudo de petróleo del tipo ligero. 


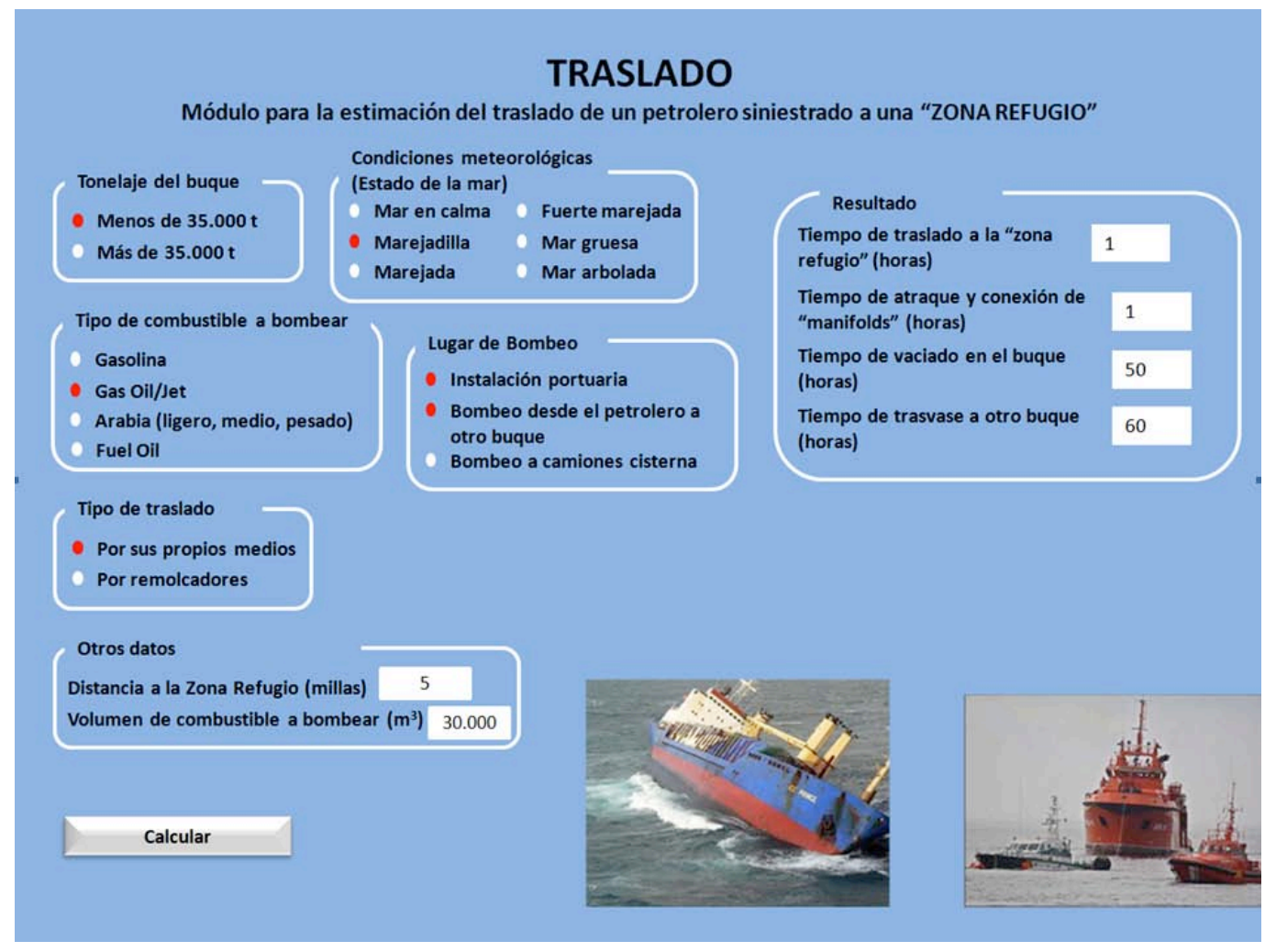

Fig. 3 Pantalla de entrada y salida de datos de una simulación con el modelo "REFUGIO".

La simulación aportó los siguientes resultados: el tiempo de traslado a la zona refugio se estima en 1 hora, el tiempo de atraque y conexión de los tubos distribuidores a la terminal de descarga igualmente de 1 hora y el tiempo de trasvase de los hidrocarburos a una terminal portuaria de 50 horas.

En el caso de que no se aconsejase trasladar el buque siniestrado a una zona refugio y se decidiese trasvasar los hidrocarburos a otro buque, el tiempo estimado de dicha operación sería de unas 60 horas.

Para el caso de no ser aconsejable el traslado del petrolero siniestrado a una zona refugio el modelo calcula el tiempo necesario para efectuar el trasvase de los hidrocarburos a un buque almacén.

Aún en el caso de decidirse trasladar un petrolero siniestrado a una zona refugio sería conveniente contemplar la posibilidad de que la carga del petrolero se derramase en el mar. Bajo esta hipótesis se puede estimar los costes derivados de la limpieza y restauración del entorno costero que fuese afectado por el vertido de hidrocarburos.

Para dicho estudio, es necesario determinar el número de personas que van a participar, no considerando voluntarios, sino personal entrenado y preparado para dichas tareas de limpieza y restauración de la costa. Dentro de éste número, es necesario determinar una jerarquía, ya que para una correcta organización es necesario tener muy claro el papel de cada uno de los participantes. Dentro de los costes, se deberá considerar los días necesarios para recuperar la costa, los sueldos del personal implicado, los costes de manutención, los costes de transporte y alojamiento (en el caso de ser necesario) y el coste de los equipos de protección individual. La Fig. 4 muestra el resultado de una simulación mediante la cual se han estimado los costes de limpieza y restauración de un entorno costero de la Bahía de Alcudia que ha sido afectado por un vertido de 5.000 toneladas de fueloil. El coste estimado es de $€ 2.764 .085$. 


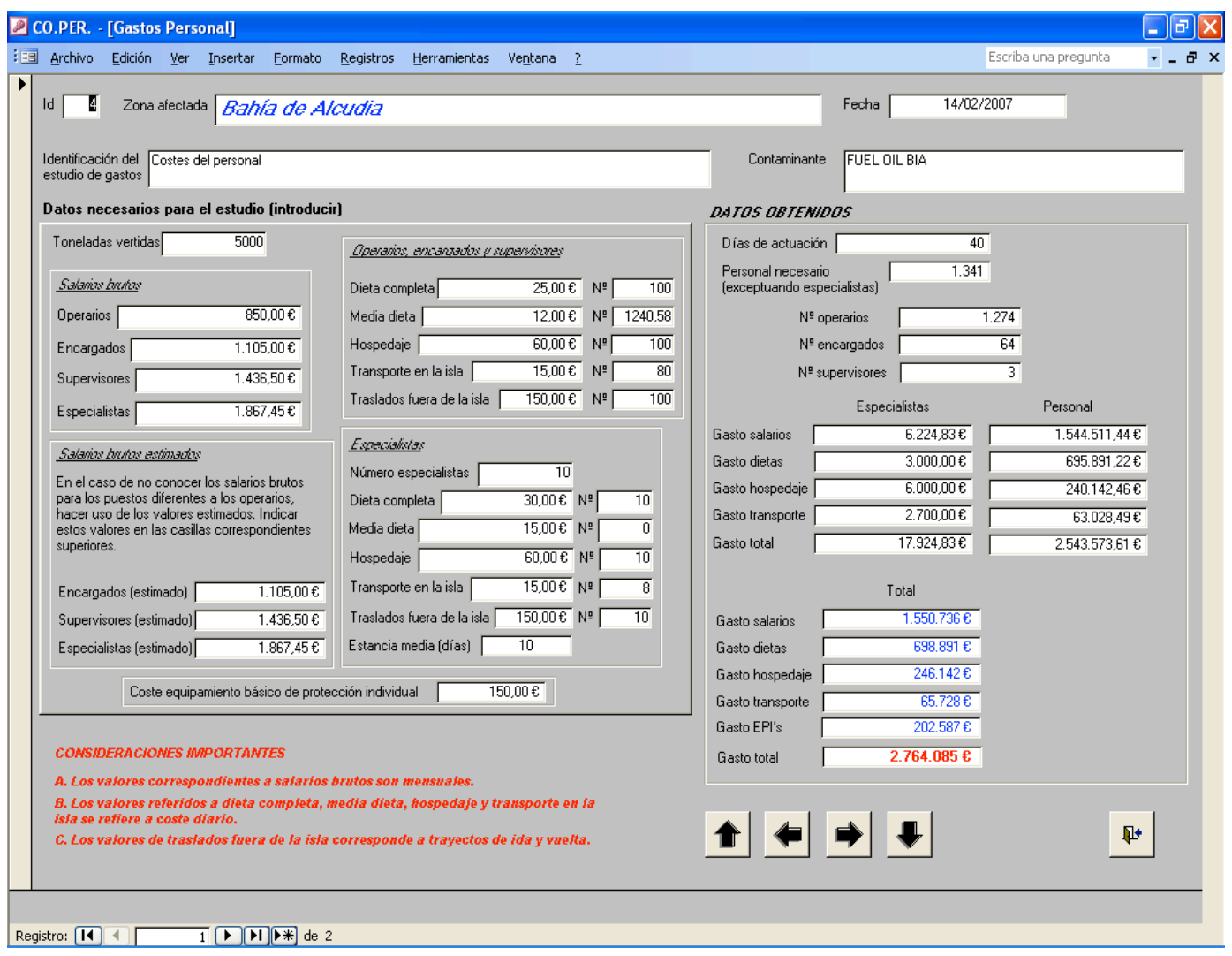

Fig. 4 Pantalla de entrada y salida de datos del coste de limpieza de un entorno costero de la Bahía de Alcudia afectada por un vertido de 5.000 toneladas de fueloil.

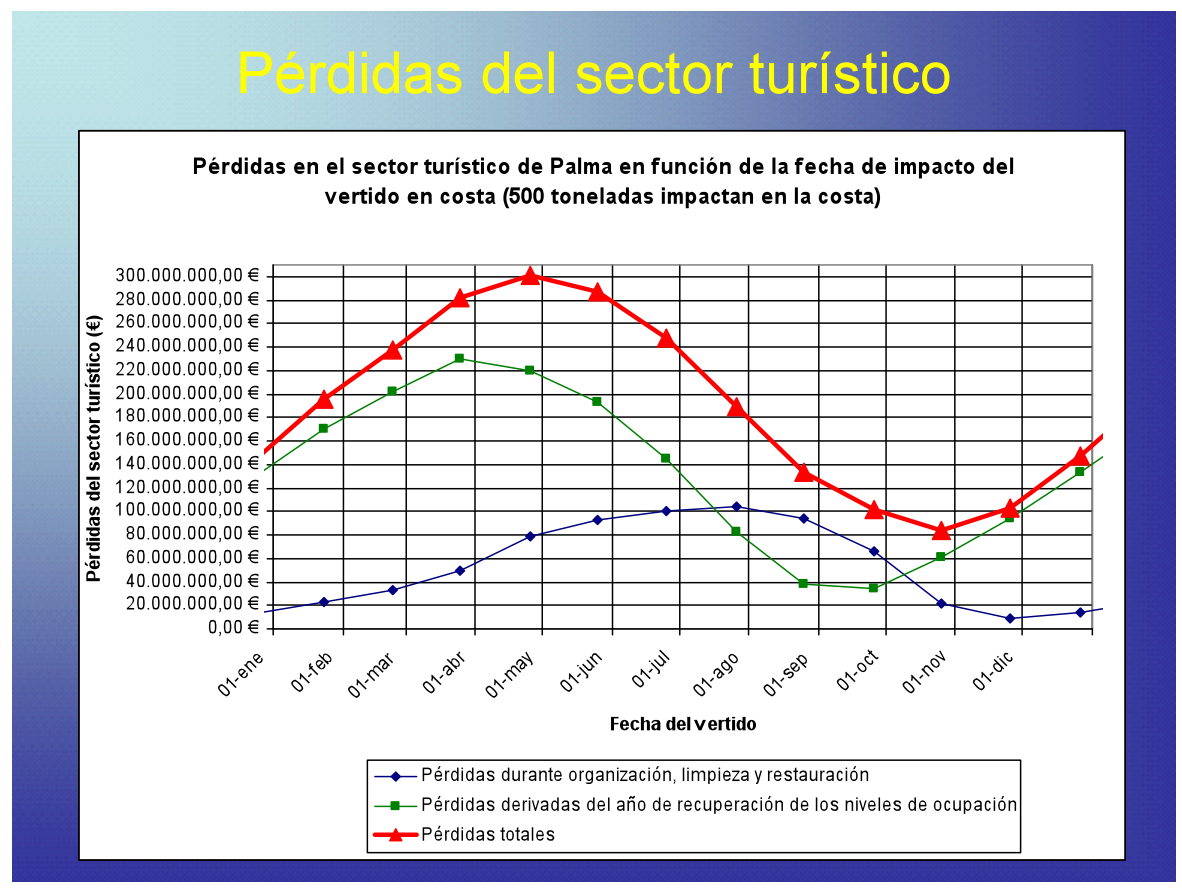

Fig. 5 Pantalla de salida de datos de las pérdidas que en el sector turístico puede originar un vertido de 500 toneladas de fueloil. 
Igualmente es necesario estimar las pérdidas que en el sector turístico puede originar un vertido de hidrocarburos, para diferentes fechas. La Fig. 5 muestra las pérdidas que en el sector turístico de Palma de Mallorca puede originar un pequeño vertido de 500 toneladas de fueloil. Dicho esquema muestra, además de las pérdidas totales, las pérdidas durante la organización, limpieza y restauración de la zona afectada, al igual que las devengadas durante el año de recuperación de los niveles de ocupación hotelera.

\section{CONCLUSIONES}

Se ha elaborado una escala que permite clasificar las zonas costeras en función de su idoneidad para ser zonas refugio, aplicándose la misma a un total de 245 costas de la isla de Mallorca, a las que en potencia podrían trasladarse petroleros siniestrados.

Se ha elaborado un programa informático mediante el cual se puede estimar el tiempo de traslado de un petrolero a una zona refugio, el tiempo de atraque y conexión a las mangueras de descarga y el tiempo de trasvase de los hidrocarburos a una terminal de descarga o a otro buque petrolero.

Del análisis de las características de las zonas costeras, de las características del propio siniestro (riesgo explosión, magnitud del derrame y tipo de hidrocarburo) y de las matrices de factores ambientales y factores infraestructurales, se puede determinar que en el caso de no existir riesgo de explosión y para vertidos fácilmente controlables, la mejor opción será conducir el petrolero siniestrado a la terminal portuaria más cercana que disponga de medios de bombeo y de almacenamiento de los hidrocarburos transportados (Puertos de Palma y Alcudia).

En el caso de que exista riesgo de explosión, se optará por zonas alejadas de grandes núcleos de población, siendo posible el desalojo rápido y controlado de la población existente. Igualmente debe ser una zona costera abrigada, que permita una fácil contención de los hidrocarburos derramados mediante barreras convencionales y adsorbentes y que cuente con acceso por carretera, para el desplazamiento de los sistemas de recuperación (skimmers y adsorbentes) y del combustible del buque a lugares de almacenamiento adecuados. Un ejemplo de este tipo de zona refugio existente en la isla de Mallorca sería el Puerto de Valldemossa.

Por la proximidad del Parque Natural del Archipiélago de Cabrera a la isla de Mallorca, se evitará, en toda la medida de lo posible, que la ruta diseñada para el traslado del petrolero siniestrado a la zona refugio se aproxime a dicho parque natural. Estas mismas medidas deberán tomarse para zonas próximas a la Albufera de Alcudia (parque natural) y S'Albufereta de Pollensa (reserva natural).

Una vez analizada toda la información inherente a las posibles zonas refugio en la isla de Mallorca se llega a la conclusión de que no existe ninguna zona que cumpla con las características del mayor nivel de valoración de la escala, por lo que se aconsejaría disponer de una zona refugio que sirviese para las Islas Baleares, ya que las mismas se encuentran en un entramado de rutas de petroleros que abastecen de crudos de petróleo a las refinerías de Tarragona, Castellón de la Plana y Cartagena, al igual que de rutas por las que circulan petroleros que transportan derivados del petróleo a todas las Islas Baleares. Así pues, y atendiendo a la importancia que tiene el turismo para las Islas Baleares, contando con aproximadamente 12 millones de turistas al año, en el caso de producirse un accidente de un petrolero y no poder trasladarlo a una zona adecuada, los efectos en la economía podrían ser catastróficos.

Se ha estimado en $€ 2.764 .085$ el coste de limpieza y restauración de un entorno costero de la Bahía de Alcudia afectado por un vertido de 5.000 toneladas de fueloil.

También se han estimado, en función de la fecha en que se produzca el vertido, las pérdidas que en el sector turístico puede originar un pequeño vertido de 500 toneladas de fueloil. Dicho cálculo muestra además de las pérdidas totales las pérdidas durante la organización, limpieza y restauración de la zona afectada, al igual que las 
devengadas durante el año de recuperación de los niveles de ocupación hotelera.

A todos estos costes habría que añadirles los derivados de la promoción turística, lo que se estima en unos 6 millones de Euros.

\section{TRABAJOS FUTUROS}

Se está completando el estudio sobre los costes que se pueden derivar de la limpieza y restauración de diferentes entornos costeros, bajo el efecto de diferentes tipos de hidrocarburos, con la finalidad de obtener un método objetivo y aplicable a cualquier zona costera.

Este estudio se está desarrollando en formato informatizado, con la finalidad de que pueda ser utilizado por cualquier gestor de un plan de contingencia, sea cual sea su localización.

Mediante un servidor en el que se centralice la información, será posible que todos los responsables de alguna de las fases del plan de contingencia puedan consultar la información, con el fin de minimizar el tiempo de respuesta.

\section{AGRADECIMIENTOS}

Los autores desean expresar su agradecimiento al Ministerio de Educación y Ciencia, Secretaría General de Política Científica y Tecnológica, por la concesión de una ayuda para la realización del proyecto TRA2004-02460 titulado: "Caracterización de zonas de refugio para buques siniestrados que transportan hidrocarburos: su aplicación a las islas Baleares".

\section{REFERENCIAS}

Bergueiro, J.R. (1993). La marea negra del Mar Egeo en La Coruña". Video (duración: 30 minutos). Universitat de les Illes Balears. Servei de Recursos Àudio-Visuals. España.

Bergueiro, J.R. \& Domínguez, F. (1997). La Gestión de los Derrames de Hidrocarburos en el Mar. Consejería de Interior del Gobierno de las
Islas Baleares. Dirección General de Protección Civil. España.

Bergueiro, J.R., Serra, F., Santos, A. \& Moreno, S. (2004). Gestión integrada de zonas costeras ante vertidos de hidrocarburos en el mar balear. Consejería de Interior del Gobierno de las Islas Baleares. Dirección General de Protección Civil. España.

Ministerio de Medio Ambiente, "Guía de Playas (Isla de Mallorca)". Disponible en: http://www.mma.es/secciones/acm/aguas_marin as_litoral/guia_playas/baleares/mallorca_prov.ht m [consulta: 20 de Mayo de 2011].

Normativa Europea RED NATURA 2000-LIC. Disponible en: http://www.mapa.es/es/desarrollo/pags/natura20 00/natura2000.htm [consulta: 20 de Mayo de 2011].

OMI, Resolution A.949(23), Guidelines on Places of Refuge for Ships in Need of Assistance. Disponible en: www.imo.org/includes/blastDataOnly.asp/data_i d=9042/949.pdf [consulta: 20 de Mayo de 2011].

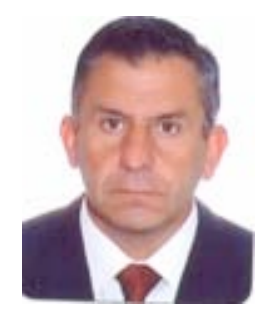

José Ramón Bergueiro. Doctor en Ciencias Químicas. Profesor Titular de Ingeniería Química de la Universidad de las Islas Baleares. $\mathrm{Ha}$ publicado 105 trabajos de investigación, presentado 110 comunicaciones a congresos, publicado 8 libros y 15 capítulos de libros sobre la "Contaminación marina por hidrocarburos". 